\title{
Stable behavioral and neural responses to thermal stimulation despite large changes in the Hydra vulgaris nervous system
}

Constantine N. Tzouanas ${ }^{1,4}$, Soonyoung Kim ${ }^{2,4}$, Krishna N. Badhiwala ${ }^{1}$, Benjamin W. Avants ${ }^{2}$, Jacob T. Robinson ${ }^{1,2,3^{*}}$

1. Department of Bioengineering, Rice University, 6100 Main Street, Houston, Texas 77005, USA.

2. Department of Electrical and Computer Engineering, Rice University, 6100 Main Street, Houston, Texas 77005, USA

3. Department of Neuroscience, Baylor College of Medicine, One Baylor Plaza, Houston, Texas 77030, USA

4. These authors contributed equally.

*To whom correspondence should be addressed: jtrobinson@rice.edu, 713.348.2933 (J.T.R.)

\begin{abstract}
Many animals that lose neural tissue due to injury or disease have the ability to maintain their behavioral abilities by regenerating new neurons or reorganizing existing neural circuits. However, most small model organisms used for neuroscience like nematodes and flies lack this high degree of neural plasticity. These animals often show significant behavioral deficits if they lose even a single neuron. Here we show that the small freshwater cnidarian Hydra vulgaris can maintain stable sensory motor behaviors even after losing half of the neurons in its body. Specifically, we find that both the behavioral and neural response to a rapid change in temperature is maintained if we make their nervous system roughly $50 \%$ smaller by caloric restriction or surgery. These observations suggest that Hydra provides a rich model for studying how animals maintain stable sensory-motor responses within dynamic neural circuit architectures, and may lead to general principles for neural circuit plasticity and stability.
\end{abstract}

\section{Significance Statement}

The ability of the nervous system to restore its function following injury is key to survival for many animals. Understanding this neural plasticity in animals across the phylogenetic tree would help reveal fundamental principles of this important ability. To our knowledge, the discovery of a set of neurons in the jellyfish polyp Hydra vulgaris that stably support a response to thermal stimulation is the first demonstration of large-scale neural plasticity in a genetically tractable invertebrate model organism. The small size and transparency of Hydra suggests that it will be possible to study large-scale neural circuit plasticity in an animal where one can simultaneously image the activity of every neuron. 


\section{Introduction}

One remarkable feature of the nervous system is plasticity - the ability to alter or reorganize neural circuits to gain or restore function. In mammals, individual neurons can alter their excitability based on presynaptic transmission frequencies [1] to maintain stable activity levels. Deficits in this process of homeostatic plasticity have been associated with neural disorders [2]. Similarly, synaptic plasticity allows collections of neurons to remap connections to produce behavioral compensation after broad injuries such as strokes, using molecular mechanisms similar to those involved in neural development [3]. Despite these insights, general principles for how neural plasticity helps to preserve behavioral function are not well known across phylogeny.

Small invertebrates would be ideal model systems for studying the processes associated with neural plasticity and behavior because they have relatively small nervous systems that make it easier to track neural circuit reorganization in living animals. However, most invertebrates show modest regeneration or lack well-developed transgenic techniques. For instance, even weeks after nerve lesions, Aplysia shows incomplete recovery of the number of large axons in nerve cores [4], receptive fields [5], and behaviors like head waving during feeding [6] and tail-elicited siphon-withdrawal reflex [7]. Neurons in the central nervous system of Drosophila do not regenerate following axotomies in either whole-brain cultures [8] or in larvae [9]. In C. elegans, axons of select neurons can regenerate after being severed [10]; however, growth trajectories are error-prone [11] even when identical neurons across animals are subjected to similar injury protocols [12]. Exceptional neural plasticity can be found in planarians, which can reform entire organisms [13] from small fragments of tissue, but these animals lack a suite of transgenic tools similar to that of Drosophila and C. elegans [14].

Hydra vulgaris is unique among invertebrate model organisms in that they are amenable to a variety of transgenic techniques and display significant neural plasticity. As examples of Hydra's remarkable regenerative capabilities, it can regrow its entire body and nervous system from a fragment as small as $\sim 300$ cells [15], [16], and its entire nervous system can be rebuilt from even a single stem cell [17]. The regenerative properties of Hydra's nervous system are due to the multipotent interstitial stem cells which continually give rise to several cell types, including the neurons. Even in an uninjured animal, there is continual turnover of differentiated neurons, with all neurons being replaced every 20 days [14]. These properties of the Hydra nervous system make it possible to study how new neurons integrate into existing circuits and how neuronal circuits are rebuilt following injury.

The small size and genetic tractability of Hydra provide additional advantages. As a millimeterscale invertebrate, an entire Hydra can be imaged at single-cell resolution [15]. Furthermore, the ability to generate transgenic lines by embryo injection [18], [19] has enabled functional calcium imaging in neurons [20] and epitheliomuscular cells [21]. In addition, cell-type specific promoters have been developed based on single-cell transcriptomic analysis of Hydra that revealed twelve neuronal subtypes, each expressing distinct sets of biomarkers linked to neuronal development and function [22], [23].

Given Hydra's potential as a model for neural plasticity, it is critical to establish behavioral assays that indicate if and when neural circuits have regained their ability to regulate behavior. While Hydra has 
been studied for over 300 years, we lack quantitative characterization of behaviors like sensory-motor responses. Studies of behavior dating back to the 1700's [24] qualitatively describe responses to light [25], chemicals [26], and temperature [27]-[29], but these experiments fall short of quantitative evaluation of behavior and neural activity. Recently, machine learning approaches have been used to identify behavioral motifs in freely moving Hydra, but these experiments have not been extended to sensory-motor responses [30].

Hydra's response to a rapid change in temperature is one sensory-motor behavior that could be used to assess neural plasticity, but it must first be precisely defined and quantified. While prior experiments suggest that Hydra can sense temperature, more work is needed to quantitatively characterize the animal's neural and behavioral response patterns to a rapid temperature change. Mast showed that when touched by heated objects, Hydra responds by bending toward the stimulus, but these experiments cannot fully distinguish between mechanosensory and thermosensory behaviors [28]. Schroeder and Callaghan reported the highest and lowest temperatures in which Hydra oligactis and Hydra pseudoligactis could survive [27]. Bosch et al. demonstrated that exposing Hydra to moderately elevated temperatures $\left(30^{\circ} \mathrm{C}\right.$ for two hours) increases their ability to survive culture at high temperatures $\left(34^{\circ} \mathrm{C}\right.$ for four days) that would otherwise be lethal [29]. These experiments show that Hydra viability depends on the ambient temperature, but they do not reveal the specific sensory-motor response to an acute thermal stimulus.

Here we show the first quantitative description of Hydra's behavioral and neural response to thermal stimulation and demonstrate that these responses are maintained even if the numbers of neurons in the animal change by a factor two due to caloric restriction or surgery. To perform this study, we developed a microfluidic device capable of delivering rapid and precise thermal stimuli without the confound of mechanical stimulation. Using this technique, we find that Hydra elongates after the onset of positive thermal stimulation, followed by a contraction lasting for the stimulus duration. Synchronous with body movements, we find temperature-dependent oscillatory neural activity within a ring of neurons in Hydra's peduncle. The frequency of this oscillation decreases for negative thermal stimulation and increases for positive thermal stimulation. We show that the frequency of the neural oscillation depends primarily on the absolute temperature of the thermal stimuli, rather than relative changes from culture baseline. Importantly, we find that these frequencies are nearly unchanged if the number of neurons in these animals change by a factor of two, whether due to caloric restrictions or surgical sectioning. These results suggest that the Hydra's nervous system is a valuable model for studying neural plasticity and robust neural circuit architectures. 


\section{Results}

Microfluidic device enables thermal stimulation and whole-body imaging of Hydra vulgaris

Because Hydra are sensitive to mechanical stimulation (e.g., touch and changes in fluid flow [31], [32]), we designed a two-layer microfluidic device to deliver thermal stimuli without mechanical confounds: a Hydra immobilization chamber fabricated above a flow layer with multiple inlet ports (Fig. 1a, see Materials and Methods). By connecting the flow layer's inlet ports to in-line heaters at different temperature setpoints, we can provide rapid and precise thermal stimulation by switching fluid flow through the different inlet ports - a process that we automate using microcontrollers (see Materials and Methods). In our experiments, one inlet (i in Fig. 1a) provides fluid that is maintained at Hydra's culture temperature to serve as a control. A second inlet provides fluid that is maintained at a stimulus temperature ranging from $9^{\circ} \mathrm{C}$ to $36^{\circ} \mathrm{C}$. To validate our ability to rapidly and repeatedly modulate the temperature of the Hydra immobilization chamber, we measured the temperature of the device glass with an IR camera (Fig. 1b-c). We found that we could switch between temperature setpoints in approximately one second, and that the temperature set points were repeatable to within a standard deviation of $0.5^{\circ} \mathrm{C}$ across multiple stimulation cycles.

Positive thermal stimulation drives sequential elongation and contraction in Hydra vulgaris

Having fabricated a microfluidic device that allows us to thermally stimulate and simultaneously image Hydra, we supplied fluids of different temperatures to the flow layer to measure how Hydra responds to a rapid change in temperature. We observed no statistically significant changes to Hydra's body length in experiments when we switched the source of the flow layer between two sources at $18^{\circ} \mathrm{C}$ compared to experiments with no fluid flow (" $18^{\circ} \mathrm{C}$ " and "No flow" curves in Fig. $2 \mathrm{C}$ ), showing the developed microfluidic device does not produce mechanical stimulation.

To avoid synchronizing thermal stimuli with natural rhythmic behavior [32], [33] or entraining a periodic response, we applied the thermal stimuli for a period of 60 seconds and randomized the time interval between successive stimuli (Fig. 2a). To quantify changes in Hydra's posture, we applied DeepLabCut [34] to automate labeling of Hydra's body width and length using hypostome (oral end), basal disc (aboral end), leftmost, middle, and rightmost points in the body column region (Fig. 2b, see Materials and Methods). We found Hydra respond to a rapid change in temperature $\left(18^{\circ} \mathrm{C}\right.$ to $30^{\circ} \mathrm{C}$ over approximately $1 \mathrm{~s}$ ) by first elongating, and then longitudinally contracting. The peak body length occurs approximately 20 seconds after the stimulus onset (Fig. 2c-d).

Subset of peduncle neurons show periodic calcium spikes in response to thermal stimulation

Using fluorescent imaging of transgenic Hydra that express the calcium-sensitive fluorophore GCaMP6s in neurons, we observed bursts of synchronous calcium spikes following thermal stimulation in a group of neurons in the animal's peduncle (Fig. 3b). We will refer to these neurons as temperature responsive (TR) neurons. To quantify this effect, we selected a region of interest that encompassed the 
entire Hydra peduncle (Fig. 3b). Although not all neurons in this region are putative TR neurons, the strong signal produced by the synchronous TR neuron activity allowed us to measure the calcium spike rate using this region of interest (ROI) (Fig. 3c-d, Supplemental Video 1-2).

We found that the calcium spike rate did not significantly change when switching between fluid reservoirs at $18^{\circ} \mathrm{C}$, suggesting that changing fluids in the flow layer did not produce a mechanical stimulation artifact (Fig. 3e, effect size $\delta=0.0025$ ). When we thermally stimulated Hydra across a wide range of temperatures both above and below their culture temperature, we observed that the TR neurons' calcium spike rate during positive thermal stimulation (i.e., heating) was significantly higher than in control experiments (compare conditions labeled " $24^{\circ} \mathrm{C}$ ", " $30^{\circ} \mathrm{C}$ ", and " $36^{\circ} \mathrm{C}$ " to the control condition of " $18^{\circ} \mathrm{C}$ " in Fig. 3c-d,f). Likewise, TR neurons' firing rate decreased during negative thermal stimulation (i.e., cooling; compare conditions labeled " ${ }^{\circ} \mathrm{C}$ " and " $12^{\circ} \mathrm{C}$ " to the control condition of " $18^{\circ} \mathrm{C}$ " in Fig. 3c-e).

We also found that the firing rate of the TR neurons during thermal stimulation depends primarily on the absolute temperature of the thermal stimulus and not on the relative increase in temperature. We found that when we increased the Hydra culture temperature from $18^{\circ} \mathrm{C}$ to $25^{\circ} \mathrm{C}$, there was no significant change in the calcium spike rate for most of the thermal stimulus temperatures. At high stimulus temperatures (i.e., $30^{\circ} \mathrm{C}$ and $36^{\circ} \mathrm{C}$ in Fig. $3 \mathrm{~g}$ ) we observed a statistically significant difference based on culture temperature, but the effect size was small $\left(\delta=0.25\right.$ at $30^{\circ} \mathrm{C}$ stimulation, $\delta=0.16$ at $36^{\circ} \mathrm{C}$ stimulation) compared to changing the stimulation temperature by a comparable amount (e.g., $\delta=0.71$ between Hydra stimulated at $30^{\circ} \mathrm{C}$ or $36^{\circ} \mathrm{C}$, but both cultured at $18^{\circ} \mathrm{C}$ ). These results support the conclusion that the calcium spike rate of TR neurons depends primarily on the absolute temperature of a thermal stimulus.

Hydra vulgaris' neural response to thermal stimulation is robust against naturally-occurring two-fold changes in neuron count

Having identified that the calcium spike rate of TR neurons encodes the absolute temperature of a thermal stimulus, we asked if this encoding is maintained even if animals have significantly different numbers of neurons. To answer this question, we first determined that the number of neurons in Hydra is linearly related to the size of the animal. Using longitudinal fluorescence imaging of Hydra over two weeks of starvation or regular feedings, we found that the number of interstitial cells (known to be comprised of $58.9 \%$ neurons, $23.8 \%$ progenitor cells, $7.8 \%$ nematocytes/nematoblasts, and $0.4 \%$ germline cells [22]) scaled with Hydra's body volume, consistent with prior reports of the density of neurons as a function of animal size [35], [36] (Fig. 4a-c).

To compare the thermal responses of animals with different sizes, we separated animals into "large" and "small" cohorts according to their body size. Based on the differences in body size and our measured relationship between body size and number of neurons, we estimate that the difference in neuron count between these groups is approximately two-fold (Fig. 4d-e). Note that while the small and large populations have a two-fold difference in the mean, the distributions do overlap due to the naturally occurring inhomogeneity in the small and large populations (Supplemental Fig. 1). Nevertheless, we 
expect that these groups would show differences in behavior if the thermal response were dependent upon the number of neurons in the animal.

When we measured the calcium spike rates during thermal stimulation, we found a nearly identical temperature dependence in both small and large animals (Fig. 4f), suggesting that Hydra maintain stable encoding of thermal stimuli despite significant, two-fold differences in the size of their neural circuits. Both groups exhibit decreased calcium spike rates during negative thermal stimulation and increased calcium spike rates during positive thermal stimulation. Although some temperatures did show statistically significant differences between the thermal response of the "small" and "large" animals (Fig. 4f), the effect size $(\delta)$ is small compared to the effect size of changes in stimulus temperature (e.g., $\delta=0.96$ when stimulating at $36^{\circ} \mathrm{C}$ as compared to culture temperature baseline; $\delta=0.21$ between large and small animals when stimulated at $\left.36^{\circ} \mathrm{C}\right)$. At $9^{\circ} \mathrm{C}$ stimulation and control temperature $\left(18^{\circ} \mathrm{C}\right)$, the effect size $\delta$ between the small and large group was 0.66 and 0.67 , respectively, which is comparable to the effect size of when comparing the calcium spike rate from the same animal measured on subsequent days $(\delta=0.61$, Supplemental Figure 1). Despite remodeling their nervous system to account for a two-fold increase or decrease in the numbers of neurons, Hydra are able to consistently encode the temperature of a thermal stimulus.

Surgical removal of half of Hydra's neurons does not significantly affect neural response to thermal stimulation

While animals with naturally-occurring large and small nervous systems showed similar responses to thermal stimuli, we also wondered if this behavioral stability would apply to individual animals that had recently lost neural tissue. To answer this question, we cut the organisms in half along their oral - aboral axis. Thanks to Hydra's radial symmetry and regenerative abilities, these surgically altered animals repaired themselves within 48 hours, yielding a full animal with roughly half the number of neurons as the original whole (Fig. 5a-C, see Materials and Methods). By measuring neural responses to thermal stimulation both before and two days after this longitudinal sectioning, we could experimentally modulate the number of neurons in an organism and quantify resultant changes in neural activity patterns (Fig. 5b). Specifically, we generated on average a two-fold decrease in neuron count between whole and bisected Hydra (Fig. 5c-d); however, we found that the neural response to thermal stimulation remained stable across all stimulus temperatures from $9^{\circ} \mathrm{C}$ to $36^{\circ} \mathrm{C}$ (Fig. $5 e$ ). Namely, we found no statistically significant changes in the calcium spike rate when comparing Hydra before and after surgical resection ( $N=5$ animals per stimulus temperature). In order to account for naturallyoccurring variability between animals, we performed bootstrapping on the calcium spike rates within size groups and calculated the corresponding effect size ( $\delta$ ) for comparison (Supplemental Fig. 3). We found that the effect sizes between the calcium spike rates of whole and bisected Hydra are negligible to small $(\delta<0.18)$ for all stimulation temperatures except for $30^{\circ} \mathrm{C}$, and moderate $(\delta=0.41)$ for $30^{\circ} \mathrm{C}$ stimulation. These effect sizes are all small compared to the day-to-day variability within an animal (Supplemental Fig. 1) and to the change in number of neurons, which has a maximum Cliff's delta of 1 
(Fig. 5d). Thus, even when we used surgical resection to create a two-fold decrease in neuronal count, Hydra maintains stable encoding of thermal stimulus temperature.

\section{Discussion}

Unlike many invertebrate model systems like C. elegans and Drosophila that have stereotyped neural architecture with well-defined numbers of neurons, Hydra's highly plastic nervous system allows us to study how behaviors like sensory-motor responses are maintained as neural circuits remodel and incorporate new neurons. The two-layer microfluidic device shown here is a well-controlled method to study this process because it allows us to simultaneously image neural activity and behavior while we deliver thermal stimuli without mechanical stimulation artifacts. Using this technology, we were able to characterize Hydra's response to rapid changes in temperature. Following thermal stimulation, we found that the animal first elongates and then contracts, with the peak body length occurring approximately 20 seconds after the stimulus onset. This behavioral response to a thermal stimulus is accompanied by synchronous periodic activity in a group of neurons near the animal's aboral end that we refer to as the TR neurons. The frequency of TR neuron activity (defined as calcium spike rates) depends on the absolute temperature of the thermal stimulus, as opposed to relative changes from the baseline culture temperature. This encoding is maintained even if the number of neurons in the animal changes by a factor of two via either natural changes to the animal's size or by surgical resection along the oral-aboral axis.

One remaining question is the identity of the TR neurons. Their location near the animal's foot suggests that they may be the contraction burst (CB) neurons that are associated with animal contractions [20], but more work is needed to determine if TR neurons and CB neurons are a completely overlapping neuronal subtype. This could be answered using cell type-specific promoters or other cell type labeling strategies that can be developed based on recent single-cell transcriptomic data. Among the 12 neuronal subtypes, the neurons in the peduncle have been identified to be comprised of ectodermal ganglion neurons that express Hym-176A, Hym-176C and Hym-176D, and ectodermal sensory and ganglion neurons expressing RFamide preprohormone $A$ [22]. Considering Hym-176C and $H y m-176 D$ are only present in the peduncle, modulating neurons expressing those genes can help us get one step closer to understanding the identity of the TR neurons.

Another question is how the TR neurons maintain their thermal response properties in circuits of different sizes. It is possible that Hydra have homeostatic mechanisms to maintain a neural circuit architecture that has the same thermal response even as neurons are added and removed. It is also possible that the thermal response circuit is simply not affected by the overall numbers of neurons in the circuit, provided there is a critical minimum number of each constituent cell type. Alternatively, the TR response may be a cellular property that is independent of the circuit size and architecture (e.g., harnessing synaptic scaling or intrinsic homeostatic plasticity to maintain the activity set point [37]). The answer to this question could be elucidated through single cell electrophysiology, blocking cell-to-cell signaling in this circuit, or using cell-type specific promoters to alter properties of neuronal subsets. 
Hydra's combination of extensive neural plasticity and promise for genetically targeted manipulations could help evaluate the relative contributions of cellular and ensemble-level properties to this circuit and its stability.

By studying sensory-motor transformations like this thermal response, we may also learn about fundamental properties of neural circuits that are common to many species. For example, the TR neurons show characteristics of a neural oscillator, which is a common motif across phylogeny. Such an approach could point toward conserved principles of neural circuits that appeared early in our evolutionary history.

Overall, the ability to study neural and behavioral responses to thermal stimulation in a highly regenerative animal amenable to cellular-resolution fluorescent imaging offers many advantages as a model system for uncovering how neural circuits remodel without compromising their function.

\section{Materials and Methods}

\section{Microfluidic device fabrication:}

All microfluidic devices were fabricated using polydimethylsiloxane (PDMS) (Sylgard 184). The thermal stimulation chip is a two-layer device: bottom stimulus flow channels and a top Hydra immobilization chamber, separated by a glass coverslip. By separating Hydra from fluid flow, the animal can be rapidly heated/cooled (by flowing water at controlled temperatures through the bottom stimulus flow channels) without undesired mechanical stimulation from high flow rates (avoided due to the glass coverslip separation). To construct the double layer chip, we first fabricated the top immobilization layer by molding PDMS from a master mold adapted from a $2 \mathrm{~mm}$ diameter x $100 \mu \mathrm{m}$ tall chemical perfusion chip (designed as previously described) [38]. Briefly, a circular observation chamber designed specifically for Hydra was patterned using soft lithography on a silicon substrate, and a $\sim 5 \mathrm{~mm}$ thick layer of PDMS was molded from this master mold. After punching holes for the inlet and outlet ports with a $1.5 \mathrm{~mm}$ diameter biopsy punch, the immobilization layer was $\mathrm{O}_{2}$ plasma bonded to a $12 \mathrm{~mm}$ diameter glass coverslip.

This top immobilization chip was then placed with a glass coverslip side facing down directly on the center of the master mold for the flow layer. Uncured PDMS was poured into the flow layer mold surrounding the immobilization chamber, taking care not to clog the ports in the immobilization layer. The master mold for the flow layer (adapted from Duret et al [39]) was 3D-printed with 1mm tall channels (Form 2, Flexible Resin, Formlabs). After curing the PDMS for the bottom flow layer (thereby embedding the immobilization chamber), holes were punched for inlet/outlet access for the flow layer. Finally, this double layer microfluidic chip was $\mathrm{O}_{2}$ plasma bonded to a $500 \mu \mathrm{m}$ fused silica wafer (University Wafer).

After unloading Hydra from the device at the end of a trial (see "Loading and unloading Hydra"), both layers of the microfluidic device were rinsed with Hydra media (media adapted from the laboratory of Robert Steele), sonicated in Hydra media for at least 10 minutes, and soaked at room temperature in Hydra media. Such cleaning allowed for devices to be reused across multiple days of trials. 


\section{Hydra strains and maintenance:}

All trials were conducted on transgenic lines with neuronal expression of GCaMP6s, developed with embryo microinjections by Christophe Dupre in the laboratory of Rafael Yuste. Hydra were cultured using the protocol adapted from the laboratory of Robert Steele (UC Irvine). Hydra were raised at either $18^{\circ} \mathrm{C}$ or $25^{\circ} \mathrm{C}$ in incubators, both with $12: 12$ hours of light:dark cycle. Animals were fed freshly hatched artemia nauplii three times a week and cleaned after approximately 4 hours with Hydra media. All animals used in trials were starved for a day prior to thermal stimulation experiments and were not reused.

\section{Loading and unloading Hydra:}

Hydra were loaded into the inlet port of Hydra enclosure using a $10 \mathrm{~mL}$ syringe with attached tygon tubing. Hydra were then pulled a couple of centimeters into the tygon tubing before the tubing was inserted into the inlet port of the microfluidic device. By applying gentle pressure and gently pulsing on the plunger of the syringe, Hydra could be successfully loaded into the immobilization chamber without damage from mechanical shear. After the experiments, Hydra was removed from the device by applying pressure on the plunger of a tygon tubing-attached syringe and flushing the organism out the outlet port.

\section{Thermal stimulation assay:}

After loading Hydra, two programmable syringe pumps (New Era NE-500) controlled by an Arduino Mega ADK were used to drive the flow of deionized water at a rate of $6 \mathrm{~mL} / \mathrm{min}$ through two inlet ports of the thermal stimulation device. The third inlet port was connected to an additional syringe (useful for removing air bubbles when initially filling the bottom stimulus flow channels prior to the start of a trial), and the device outlet port was connected to a water collection container. Fluid flow from the two pumps was heated/cooled using two in-line heaters (SC-20, Warner Instruments) regulated by a Dual Channel Bipolar Temperature Controller (CL-200A, Warner Instruments). One in-line heater supplied Hydra's culture temperature during control periods (i.e. $18^{\circ} \mathrm{C}$ or $25^{\circ} \mathrm{C}$ ), while the other heater supplied the desired stimulus temperature of a given trial (i.e. $9^{\circ} \mathrm{C}, 12^{\circ} \mathrm{C}, 18^{\circ} \mathrm{C}, 24^{\circ} \mathrm{C}, 25^{\circ} \mathrm{C}, 30^{\circ} \mathrm{C}$, or $36^{\circ} \mathrm{C}$ ). To compensate for heat exchange between water flowing through inlet tubing and the environment, a FLIR ONE thermal camera was used to calibrate the relationship between in-line heater temperature settings and actual temperatures of the thermal stimulation device.

Each thermal stimulation trial began with two minutes at Hydra's culture temperature. Subsequently, the trial alternated between stimulus periods (single temperature for a given trial; $9^{\circ} \mathrm{C}$, $12^{\circ} \mathrm{C}, 18^{\circ} \mathrm{C}, 24^{\circ} \mathrm{C}, 25^{\circ} \mathrm{C}, 30^{\circ} \mathrm{C}$, or $36^{\circ} \mathrm{C}$ ) and control periods (based on Hydra's culture temperature; $18^{\circ} \mathrm{C}$ or $25^{\circ} \mathrm{C}$ ). Stimulus periods always lasted for 60 seconds, while control periods varied in length between 30 and 90 seconds in 15 second increments, to help distinguish between stimulus-evoked responses and spontaneous activity. The lengths of control periods were randomly ordered and averaged to 60 seconds over the course of a trial. For a visual representation of thermal stimulation protocols, see Fig. 3a. The timing of transitions between stimulus and control periods were recorded 
through the Arduino's Serial Monitor (57600 baud rate) and used to determine portions of recordings corresponding to stimulus and control periods. For experiments on size comparison of Hydra cultured at $18^{\circ} \mathrm{C}, 3$ large and 3 small animals for each temperature $\left(9^{\circ} \mathrm{C}, 12^{\circ} \mathrm{C}, 18^{\circ} \mathrm{C}, 24^{\circ} \mathrm{C}, 30^{\circ} \mathrm{C}\right.$, and $\left.36^{\circ} \mathrm{C}\right)$, 36 animals in total were used over the course of 15 days. For Hydra cultured at $25^{\circ} \mathrm{C}, 3$ animals were used at $18^{\circ} \mathrm{C}$, and 4 animals at $25^{\circ} \mathrm{C}, 30^{\circ} \mathrm{C}$, and $36^{\circ} \mathrm{C}$ over the course of 7 days.

Fluorescence imaging was conducted on a Nikon SMZ18 stereomicroscope with a SHR Plan Apo 2x objective (0.3 NA) and Andor Zyla 4.2 (16 fps, 3x3 image binning). Excitation was provided by a X-Cite Xylis XT720L at 50\% intensity through a Chroma EGFP filter cube (catalog no. 49002). The start of fluid flow using Arduino and the start of recording using Andor Solis were manually synced with a maximum delay less than 1 second.

\section{Measuring Day-to-Day Variability in Calcium Spike Rate}

To better understand the source of variability in our data we asked how much of the difference in calcium spike rates could be explained by day-to-day variability in the same animal. To answer this question, we stimulated the same whole Hydra twice at $36^{\circ} \mathrm{C}$ over a 48 hours interval ( $\mathrm{N}=3$ organisms) - the same time interval between the whole and partial Hydra experiments. We found that while one animal maintained its spike rate with no statistically significant difference, two animals had a statistically significant decrease in spike rate, even when stimulated at the same temperature before and after the 48 hour difference (Supplemental figure 2b). Accompanying effect sizes measured using Cliff's delta ( $\delta$ ) ranged from 0.04 for first animal to 0.41 and 0.61 for the latter two (overall pooled effect size of 0.35 ), contextualizing the relative magnitudes of observed experimental effects against typical day-to-day variability in Hydra.

\section{Longitudinal imaging of the entire nervous system}

Transgenic Hydra vulgaris AEP expressing GFP (green fluorescent protein) in their interstitial cell lineage were used to investigate how the number of neurons varies with animal size and nutrient availability. Animals with large body size ( $>2 \mathrm{~mm}$ long) at the beginning of the study were starved for the duration of the experiment. A control group of animals with small body size $(<1 \mathrm{~mm})$ at the beginning of the study were fed with an excess of freshly hatched artemia nauplii three times a week. Size of the animal was defined as the length of the animal in a relaxed state (between fully contracted and fully elongated). Hydra media was replaced daily for all animals.

Volumetric imaging was performed using a confocal microscope (Nikon TI Eclipse) with animals immobilized in chemical perfusion microfluidic chips (see Badhiwala et al. [38]) and chemically paralyzed with 1:5000 linalool. Animals were imaged three times a week (one day after the control animals were fed) over two weeks. All images were acquired with a 10x DIC objective. The majority of the images were acquired with $1024 \times 1024$ pixels $(x, y)$ resolution and $5 \mathrm{~mm}$ z-resolution. A lower resolution of 512 $x 512$ pixels and $10 \mathrm{~mm}$ z-resolution were used in a few cases to speed up volumetric imaging where micromotions of the animals could not be completely eliminated with chemical anesthetic (becoming increasingly important with animals starved for extended periods of time). 
To quantify the total number of neurons, a maximum intensity projection image was generated from the z-stacks. After binarizing the resulting image with a user-defined threshold, individual regions (or neurons) were summed to determine the total number of neurons. However, due to anatomical overlap of multiple neurons in high-density regions such as the peduncle, future work will focus on developing transgenic animals that express fluorescent reporters in the nuclei of specific neural cell types, rather than in the cytosol of interstitial cell derived lineages (as in the animals used here).

To quantify the body size during an experiment, we binarized the maximum intensity projection image with a lower threshold than above. Filling holes in the binarized image produced the binary mask for the whole animal. Body size was calculated as the area of this binary region, and body volume was body size times the thickness of the immobilization chamber $(160 \mu \mathrm{m})$.

The duration of the experiment was kept under two weeks, as well-fed animals can reproduce asexually by budding every 3-4 days. In fact, one of the animals in the well-fed group began forming a bud on day 10 of the experiment. Additionally, as animals are starved, they become smaller and more transparent, making them difficult to handle. In our starved group, we 'lost' one of the animals on day 15 as it was either too transparent or had shrunk considerably to not be discerned from the plate.

\section{Creating bisected Hydra}

For the purpose of investigating whether the number of neurons affect the neural response to thermal stimulation in Hydra, the animals were cut along the oral - aboral axis in order to ensure we have a significantly different distribution of body size while retaining tentacles, the hypostome, body column, and peduncle. Whole, uncut Hydra were imaged on day 1 as the same protocol described above. Then each Hydra was cut along the oral-aboral axis under a dissection microscope using an $\mathrm{X}$ acto knife. Any form of anaesthesia was not used in order to eliminate chemical perturbation in muscle or neural activities. The cut animals were incubated in separate 24-well compartments and were fed the day after being cut (day 2) to keep the feeding schedule consistent. After letting them recover for 48 to 50 hours, they were thermally stimulated again under the same condition they were exposed to before being cut. Five animals were used for each stimulation temperature. For the comparison of neural response between whole and bisected Hydra, only one of the two bisected Hydra was used.

\section{Behavioral analysis}

DeepLabCut [34] along with custom MATLAB code was used to quantify Hydra's behavioral responses to thermal stimuli. 20 frames per video were extracted for manual tracking according to kmeans clustering on DeepLabCut. Hydra in each frame was then manually annotated with the locations of the basal disc (aboral end), left side, center, and right side of the body, and hypostome (oral end). Two corners of the immobilization chamber were additionally annotated, providing a known distance (2 $\mathrm{mm}$ ) to convert pixel measurements to micrometer measurements. The annotated dataset was used as training data for DeepLabCut. After evaluating the model, the videos were analyzed using the trained model which yielded the coordinates of the seven points listed above for every frame (total of 14,600 
frames) for every video, along with annotated videos. With the coordinates obtained from DeepLabCut annotations, body width was calculated as the sum of the distance from the left side to the middle of the body and the distance from the middle to the right side of the body, and body length was calculated as the sum of the distance from the hypostome to the middle of the body and the distance from the middle of the body to the basal disc (Fig. 2b, bottom panel) with a custom MATLAB code.

\section{Analysis of neural activity in the peduncle of Hydra}

To determine the timing and frequency of calcium spikes in Hydra's peduncle oscillator, fluorescence microscopy recordings from thermal stimulation trials of neuronal GCaMP6s Hydra (see "Thermal Stimulation Assay") were processed in Fiji (ImageJ) and in MATLAB. For each frame of the recording, Fiji was used to calculate the average intensity of a ROI encompassing Hydra's peduncle and aboral regions (same ROI for the entire recording). In MATLAB, this intensity trace was smoothed (5 data point span), and the built-in findpeaks function was used to determine spike locations based on their prominence in the intensity traces. Spurious spikes (e.g. from measurement noise) were eliminated by additionally testing that each identified peak was the only local maximum exceeding a threshold prominence value within a narrow window of the peak.

Raster plots were then directly produced from the times of peduncle oscillator spikes during each stimulus period and the surrounding 30 seconds at culture temperature. Peristimulus time histograms defined as calcium firing rate were calculated by counting the number of spikes across all stimulus periods at a given stimulation temperature in a 10-second sliding window, then normalizing by the number of stimulus periods and the length of the window. Instantaneous calcium spike rates for a given thermal stimulus temperature were defined and calculated as the inverse of interspike intervals during stimulus periods.

\section{Acknowledgements}

We would like to thank Dr. Christophe Dupre and Dr. Rafael Yuste (Columbia University), Dr. Celina Juliano (UC Davis) and Dr. Rob Steele (UC Irvine) for sharing transgenic Hydra lines, and Dr. Juliano for comments on the manuscript. This work was supported in part by the NSF EDGE.

C.N.T. is funded by a Fannie and John Hertz Foundation Fellowship and by a National Science Foundation Graduate Research Fellowship. K.N.B. is funded by training fellowships from the Keck Center of the Gulf Coast Consortia on the NSF Integrative Graduate Education and Research Traineeship (IGERT): Neuroengineering from Cells to Systems 1250104.

\section{Author Contributions}

C.N.T. and S.K. performed and analyzed Hydra thermal stimulation experiments. K.N.B. performed and analyzed longitudinal imaging experiments on Hydra's number of neurons as a function of body size. C.N.T., K.N.B., and B.W.A. designed, prototyped, and fabricated the microfluidic device. C.N.T., S.K., 
bioRxiv preprint doi: https://doi.org/10.1101/787648; this version posted June 17, 2020. The copyright holder for this preprint (which was not certified by peer review) is the author/funder, who has granted bioRxiv a license to display the preprint in perpetuity. It is made available under aCC-BY-NC-ND 4.0 International license.

and B.W.A. implemented microcontroller automation of thermal stimulation experiments. C.N.T., J.T.R. supervised the research. C.N.T., S.K., K.N.B., and J.T.R. co-wrote the paper. All authors read and commented on the manuscript.

\section{Conflict of Interest}

Authors declare no conflict of interest. 
a

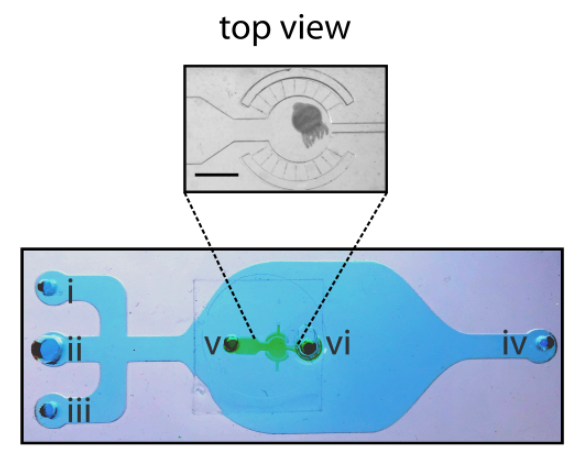

side view

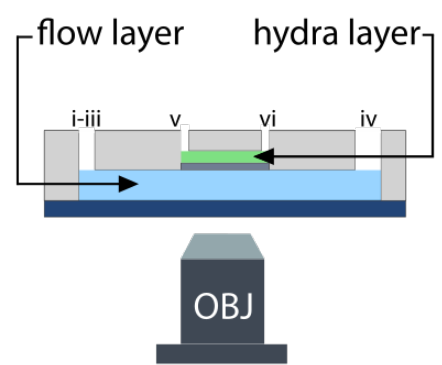

legend

i inlet port (control)

ii inlet port (stimulus)

iii air removal port

iv outlet port

$\checkmark$ hydra inlet port

vi hydra outlet port

\section{PDMS}

glass slide

glass wafer

b
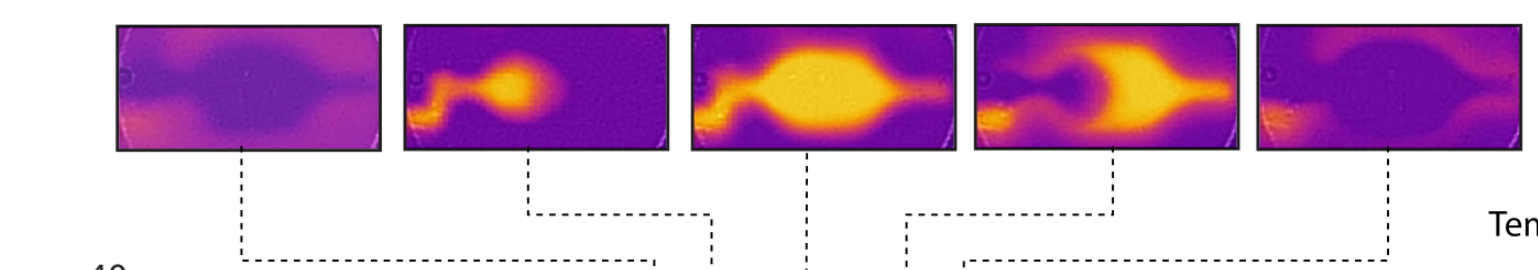

C

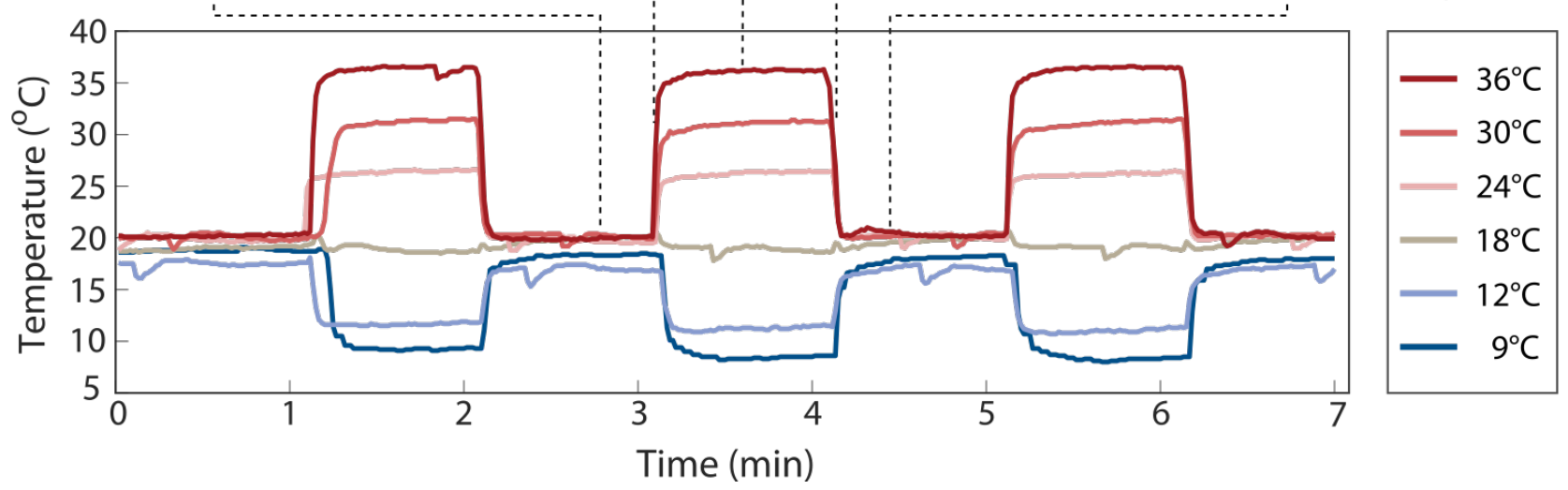

Figure 1: Characterization and validation of two-layer microfluidic device for thermal stimulation of Hydra vulgaris. a (left) Photograph of microfluidic device with blue dye in flow layer and green dye in Hydra chamber. Scale bar $=1 \mathrm{~mm}$. (middle) Schematic of cross-section of the device. (right) legend for left and middle panel. b Infrared image showing temperature of the flow layer before (leftmost), the start of (second leftmost), during (center), end of (second rightmost), and after (rightmost) thermal stimulation. c Time course of thermal stimulation at temperatures above and below Hydra's baseline culture temperature of $18^{\circ} \mathrm{C}$. 


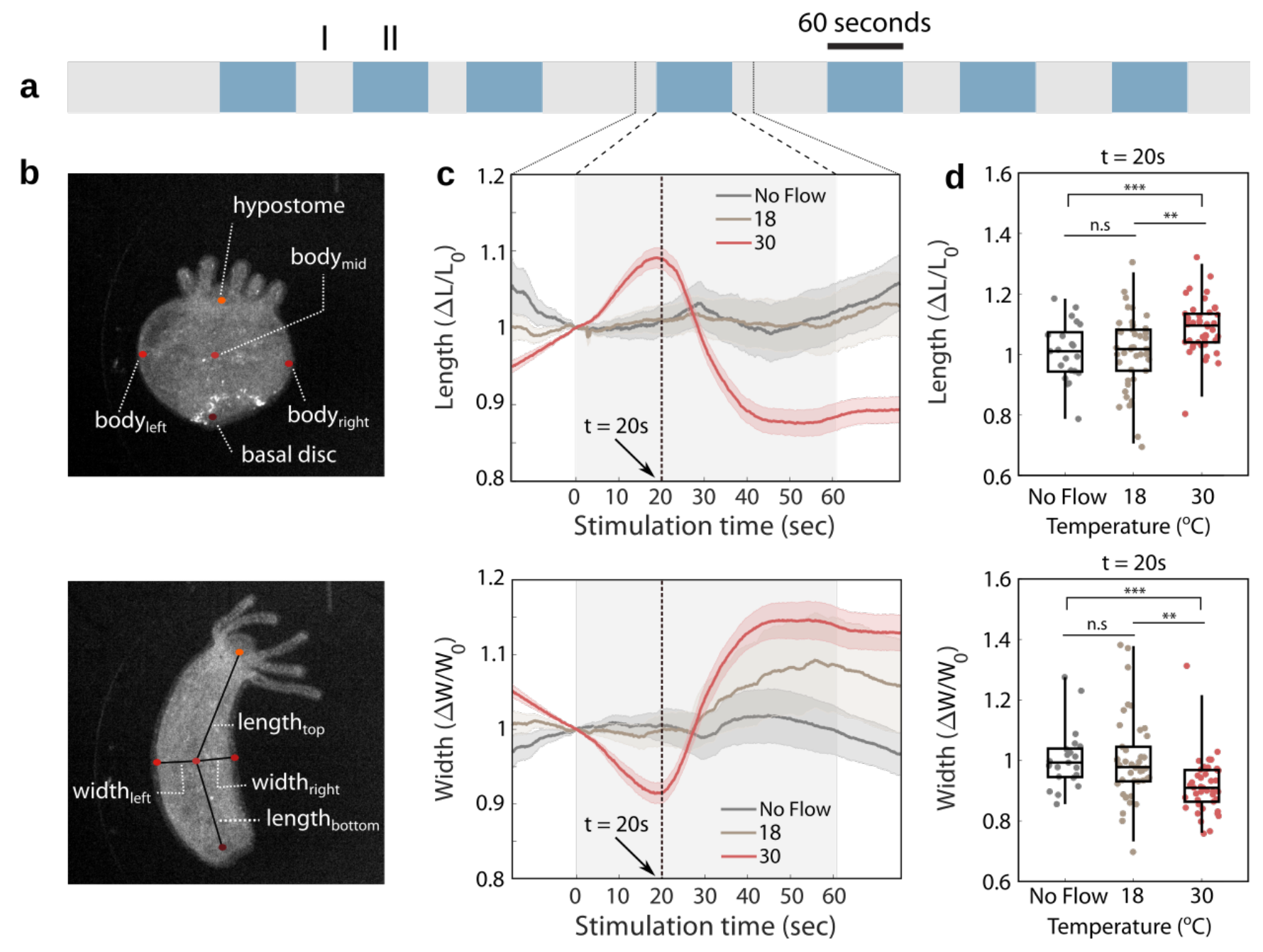

Figure 2: Hydra's body length initially increases and width initially decreases when stimulated at $30^{\circ} \mathrm{C}$. a Schematic of a representative thermal stimulation protocol. Gray regions (I) indicate nonstimulus periods at Hydra's culture temperature lasting between 30 and 90 seconds, and blue-colored regions (II) indicate stimulus periods at designated temperatures for 60 seconds. b Representative labeled frame from DeepLabCut, annotated. Top panel describes which body points were annotated, and the bottom panel shows how the length and width of the animals are determined. The hypostome is the end of the body with Hydra's mouth and tentacles (oral end), while the basal disk is used to adhere to substrates (aboral end). c Average length and width of Hydra calculated for stimulation periods and time-aligned to stimuli, with 15 seconds before and after stimulation periods. Shaded error bars correspond to standard error. $\mathbf{d}$ The change in length (top panel) and width (bottom panel) at 20 seconds after onset of stimulation, for each stimulus period $(\mathrm{t}=20 \mathrm{~s})$. $\mathrm{N}=3 \mathrm{Hydra}$ for No Flow, $\mathrm{N}=6 \mathrm{Hydra}$ for $18^{\circ} \mathrm{C}$ and $30^{\circ} \mathrm{C}$. (n.s $=$ not significant, ${ }^{* *} p<0.001,{ }^{* * *} p<0.0001$, unpaired t-test). 


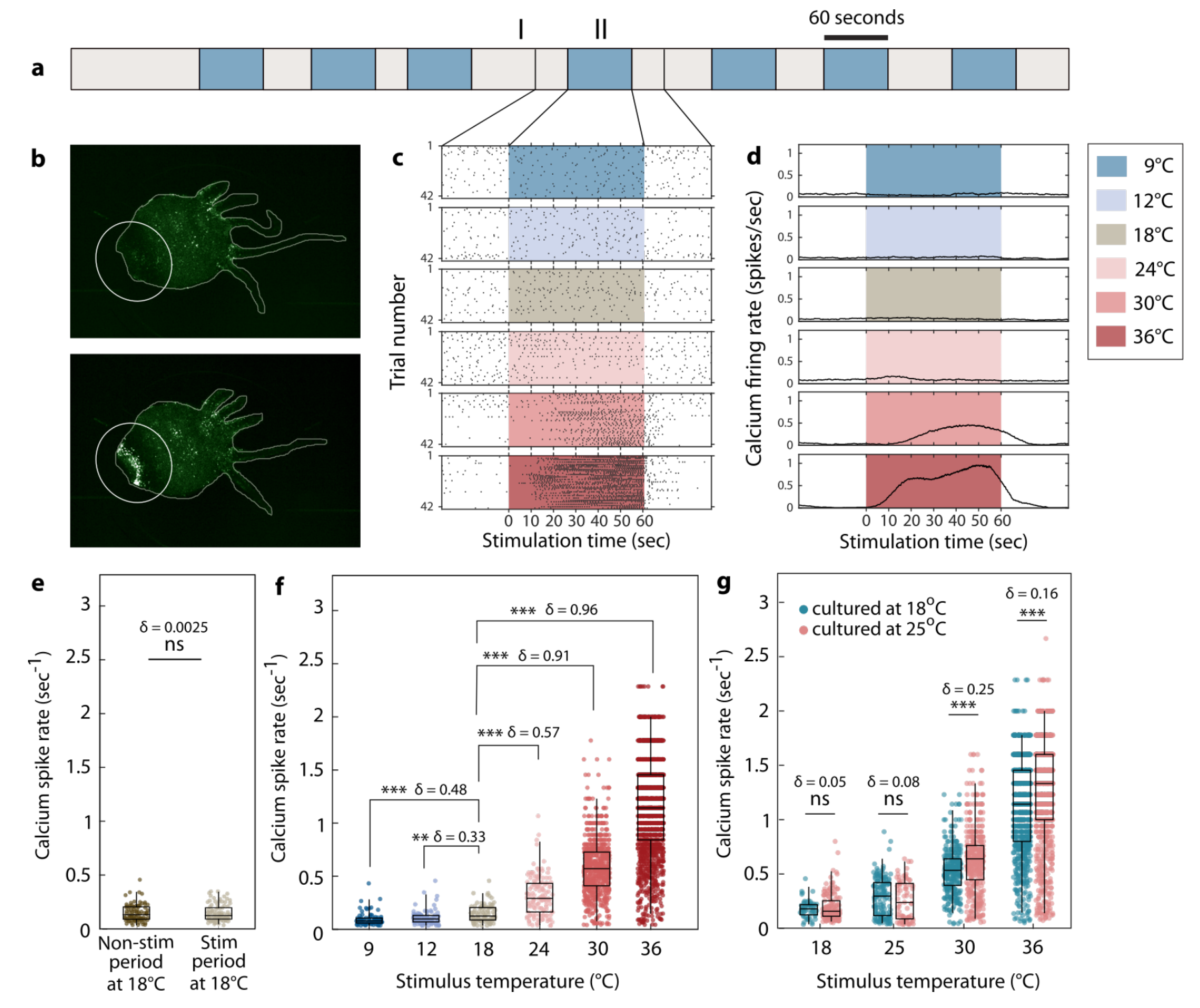

Figure 3: Thermal stimulation modulates the synchronous neural activity in the peduncle of Hydra vulgaris a Schematic of a representative thermal stimulation protocol. Gray-colored regions (I) indicate non-stimulus periods at Hydra's culture temperature (either $18^{\circ} \mathrm{C}$ or $25^{\circ} \mathrm{C}$ ) lasting between 30 and 90 seconds. The blue-colored regions (II) indicate stimulus periods at designated temperatures for 60 seconds. b Images of Hydra without synchronous neural firing (top) and during firing (bottom) in the peduncle (white circle). c Raster plot of synchronous firing events in peduncle in b. One black vertical line indicates one synchronous firing event. Each row represents the raster plot of spikes time-aligned with one stimulation (boxed regions) with 30 seconds before and after stimulation (white non-boxed region). d Peristimulus time histogram (calcium firing rate) of $\mathbf{c}$ which was calculated with a 10 second sliding window. (ns = not significant, Mann-Whitney $U$ Test. $\delta=$ Effect size using Cliff's delta) e Calcium spike rate comparison between the non-stimulation (gray-colored region I in a) and stimulation (bluecolored region II in a) periods with a stimulus temperature of $18^{\circ} \mathrm{C}$. $f$ Calcium spike rates from large and small Hydra, all cultured at $18^{\circ} \mathrm{C} . \mathrm{N}=3$ large animals; $\mathrm{N}=3$ small animals. (ns = not significant, ** $p<0.0001,{ }^{* * *} p<0.00001$, Mann-Whitney $U$ Test. $\delta=$ Effect size using Cliff's delta). g Calcium spike 
bioRxiv preprint doi: https://doi.org/10.1101/787648; this version posted June 17, 2020 . The copyright holder for this preprint (which was not certified by peer review) is the author/funder, who has granted bioRxiv a license to display the preprint in perpetuity. It is made available under aCC-BY-NC-ND 4.0 International license.

rates from Hydra cultured at $18^{\circ} \mathrm{C}$ and $25^{\circ} \mathrm{C}$. X-axis notes the stimulation temperatures. $\mathrm{N}=3$ for all animals. (ns $=$ not significant, ${ }^{* * *} p<0.00001$, Mann-Whitney $U$ test. $\delta=$ Effect size using Cliff's delta) 
a

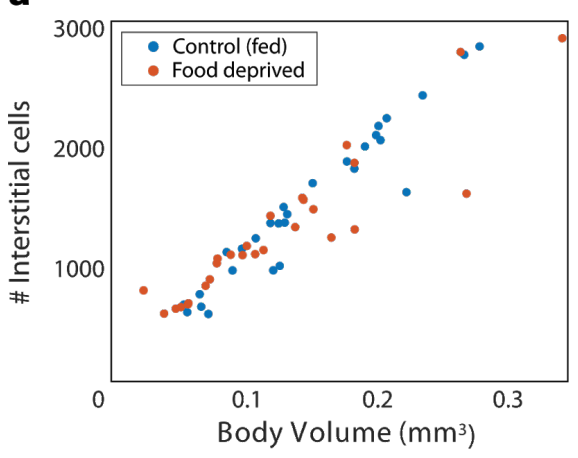

d

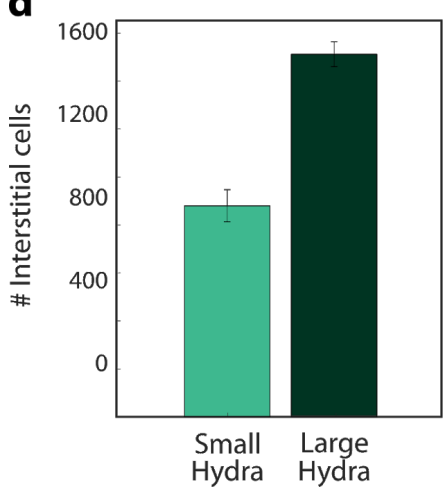

b

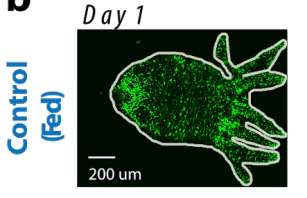

Day 1

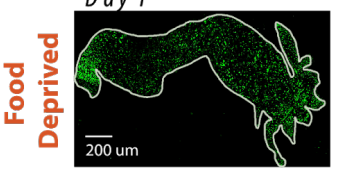

e

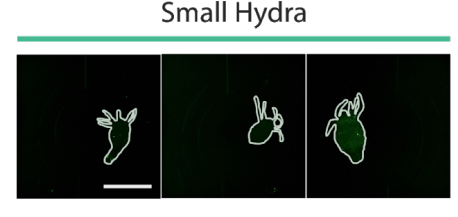

Large Hydra

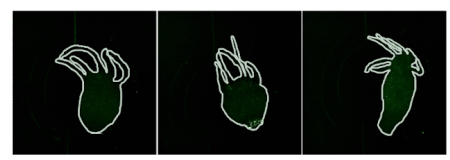

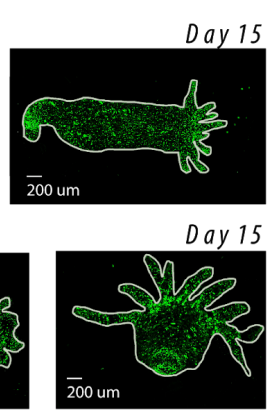

Day 15

$200 \mathrm{~cm}$
C

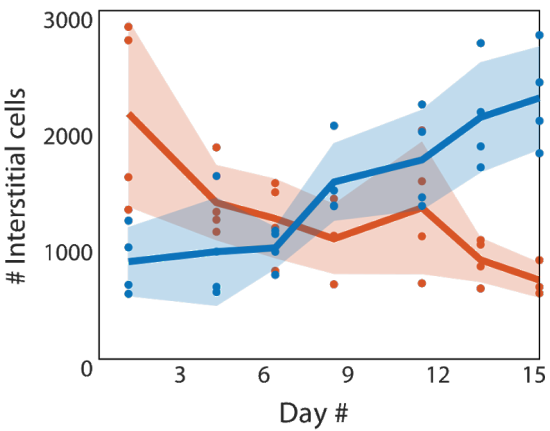

f

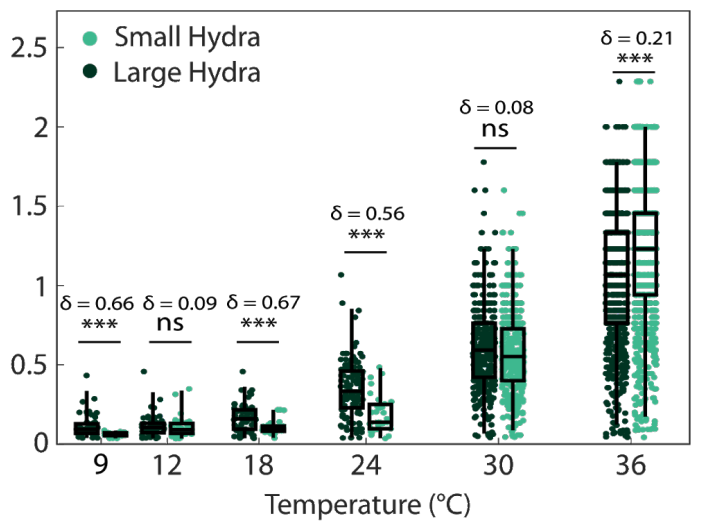

Figure 4: Frequency of thermal response neurons does not depend on the number of neurons in Hydra. a Number of interstitial cells (comprising $58.9 \%$ neurons, $23.8 \%$ progenitor cells, $7.8 \%$ nematocytes/nematoblasts, and $0.4 \%$ germline cells) as a function of animal size. b Fluorescent image of Hydra at days 1 and 15 for both control and food deprived groups (pseudo colored). c Change in the number of interstitial cells over the time course of 15 days. $d$ Estimated number of interstitial cells from measurements in a. e Three representative Hydra from small and large groups (pseudo colored) Scale bar $=500 \mu \mathrm{m}$. f Spike rates from large $(\mathrm{N}=3)$ and small $(\mathrm{N}=3)$ Hydra, all cultured at $18^{\circ} \mathrm{C}$. (ns = not significant, ${ }^{* * *} p<0.00001$, Mann-Whitney $U$ test. $\delta=$ Effect size using Cliff's delta) 
a

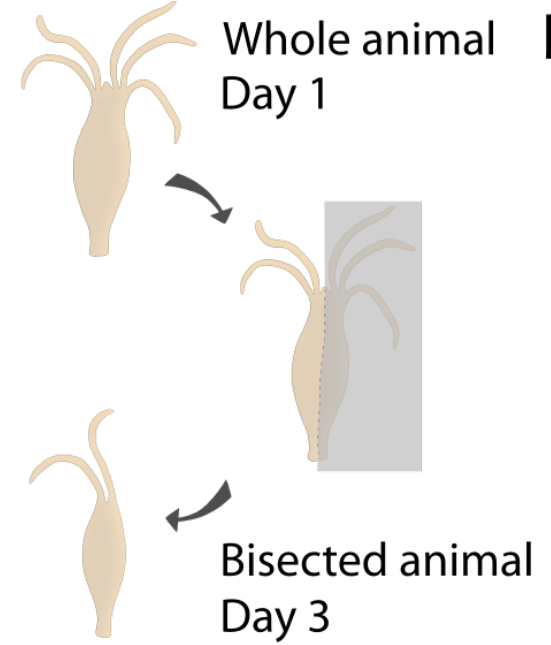

Day 3
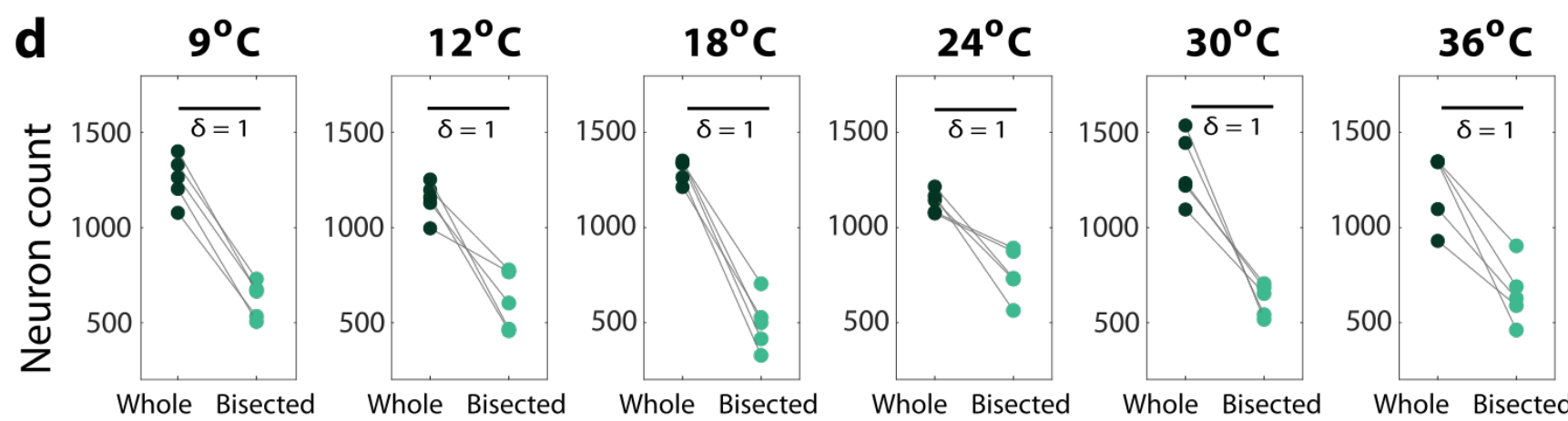

e
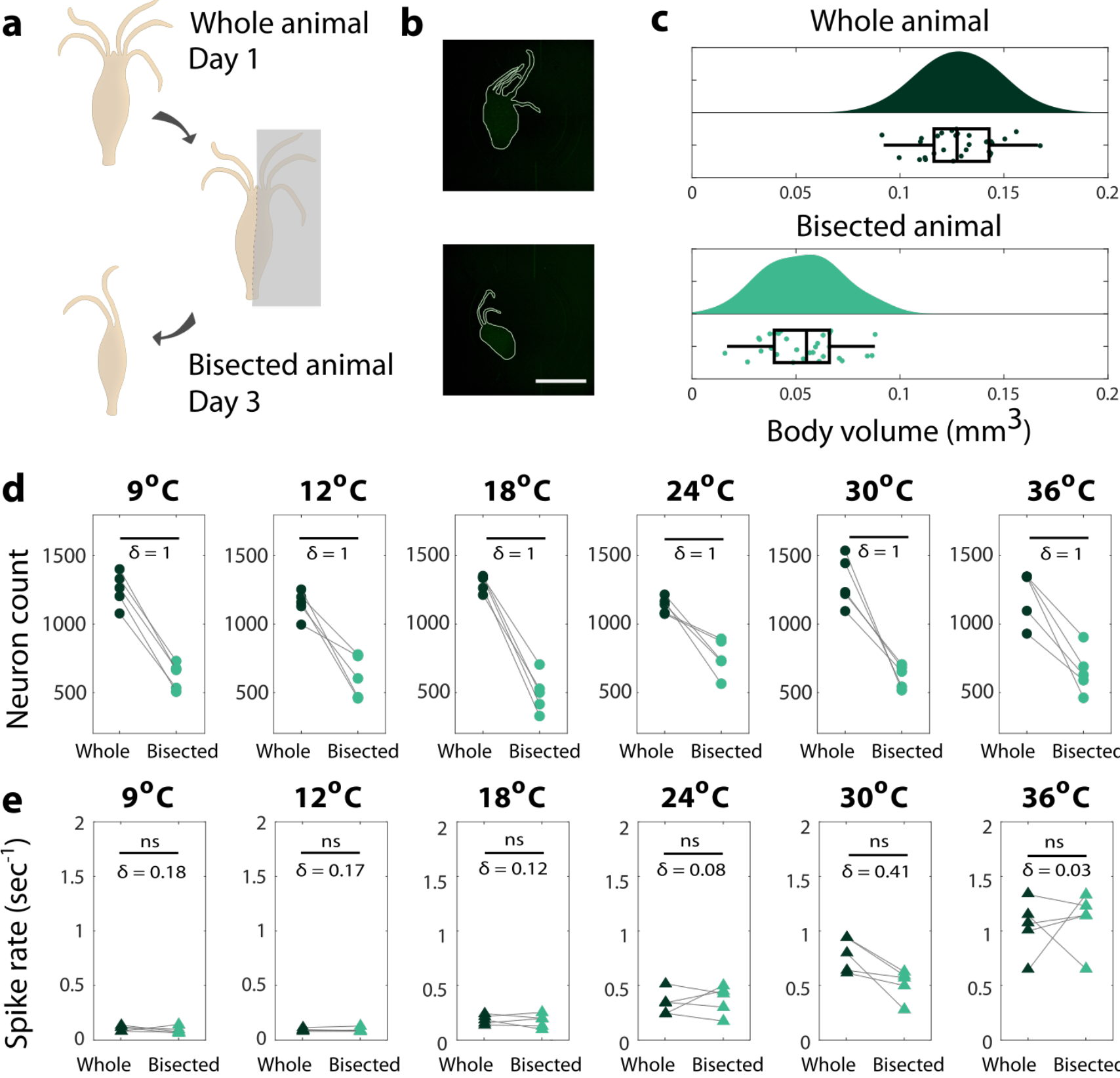

Bisected animal

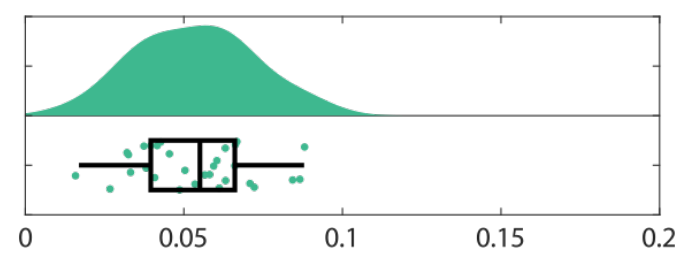

Body volume $\left(\mathrm{mm}^{3}\right)$

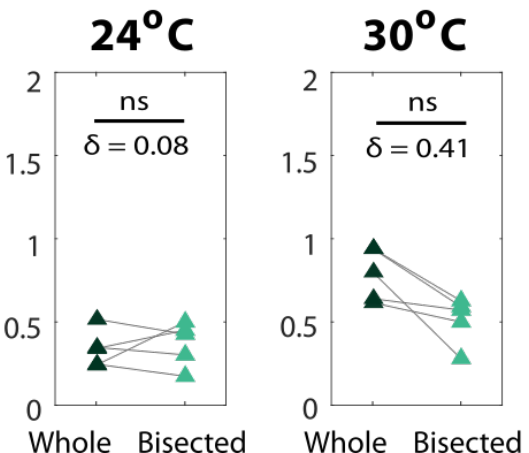

Whole Bisected

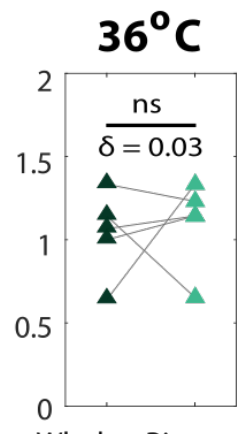

Figure 5: Neuron count within an animal does not affect neural response to thermal stimulation in Hydra vulgaris. a An illustration of the experiment. The whole animal is stimulated on Day 1 and the bisected animal is stimulated on Day 3 (48-52 hours after being cut). b Size distribution of whole and bisected Hydra. ( $\mathrm{N}=5$ for each stimulation temperature) c Representative images of whole Hydra (top panel), representative image of bisected Hydra (bottom panel). Scale bar $=500 \mu \mathrm{m}$. d Comparison of neuron count between whole $(\mathrm{N}=5)$ and bisected $(\mathrm{N}=5)$ Hydra. The dark green data points on the left correspond to the number of neurons in the whole Hydra, which are connected to the corresponding bisected Hydra, the light green points on the right. $\delta$, the effect size using Cliff's delta shown is calculated after bootstrapping with 100 iterations. e Mean spike rate comparison between whole and bisected Hydra. (ns = not significant, Wilcoxon signed-rank test. $\delta=$ Effect size using Cliff's delta) 


\section{References}

[1] G. G. Turrigiano, "The Self-Tuning Neuron: Synaptic Scaling of Excitatory Synapses," Cell, vol. 135, no. 3, pp. 422-435, Oct. 2008.

[2] V. Tatavarty et al., "Autism-Associated Shank3 Is Essential for Homeostatic Compensation in Rodent V1," Neuron, Mar. 2020.

[3] T. H. Murphy and D. Corbett, "Plasticity during stroke recovery: from synapse to behaviour," Nat. Rev. Neurosci., vol. 10, no. 12, pp. 861-872, 2009.

[4] M. L. Scott, S. C. Danzer, C. K. Govind, and M. D. Kirk, "Morphological correlates of neural regeneration in the feeding system of Aplysia californica after central nervous system lesions," $J$. Comp. Neurol., vol. 387, no. 2, pp. 279-290, Oct. 1997.

[5] M. F. Dulin, I. Steffensen, C. E. Morris, and E. T. Walters, "Recovery of function, peripheral sensitization and sensory neurone activation by novel pathways following axonal injury in Aplysia californica.," J. Exp. Biol., vol. 198, no. 10, pp. 2055 LP - 2066, Oct. 1995.

[6] I. Kupfermann, "Dissociation of the appetitive and consummatory phases of feeding behavior in Aplysia: a lesion study," Behav. Biol., vol. 10, no. 1, pp. 89-97, Jan. 1974.

[7] F. Noel, W. N. Frost, L. M. Tian, M. A. Colicos, and P. K. Dash, "Recovery of tail-elicited siphonwithdrawal reflex following unilateral axonal injury is associated with ipsi- and contralateral changes in gene expression in Aplysia californica," J. Neurosci., vol. 15, no. 10, pp. 6926 LP 6938, Oct. 1995.

[8] D. Ayaz et al., "Axonal Injury and Regeneration in the Adult Brain of Drosophila," J. Neurosci., vol. 28, no. 23, pp. 6010 LP - 6021, Jun. 2008.

[9] Y. Song, K. M. Ori-McKenney, Y. Zheng, C. Han, L. Y. Jan, and Y. N. Jan, "Regeneration of Drosophila sensory neuron axons and dendrites is regulated by the Akt pathway involving Pten and microRNA bantam," Genes Dev., vol. 26, no. 14, pp. 1612-1625, Jul. 2012.

[10] M. F. Yanik, H. Cinar, H. N. Cinar, A. D. Chisholm, Y. Jin, and A. Ben-Yakar, "Functional regeneration after laser axotomy," Nature, vol. 432, no. 7019, p. 822, 2004.

[11] Z. Wu, A. Ghosh-Roy, M. F. Yanik, J. Z. Zhang, Y. Jin, and A. D. Chisholm, "Caenorhabditis elegans neuronal regeneration is influenced by life stage, ephrin signaling, and synaptic branching," Proc. Natl. Acad. Sci., vol. 104, no. 38, pp. 15132-15137, Sep. 2007.

[12] M. Hammarlund and Y. Jin, "Axon regeneration in C. elegans," Curr. Opin. Neurobiol., vol. 27, pp. 199-207, Aug. 2014.

[13] P. W. Reddien, "The Cellular and Molecular Basis for Planarian Regeneration," Cell, vol. 175, no. 2, pp. 327-345, Oct. 2018.

[14] M. L. Scimone, L. E. Cote, and P. W. Reddien, "Orthogonal muscle fibres have different instructive roles in planarian regeneration," Nature, vol. 551, p. 623, Nov. 2017.

[15] U. Technau et al., "Parameters of self-organization in Hydra aggregates," Proc. Natl. Acad. Sci., vol. 97, no. 22, pp. 12127 LP - 12131, Oct. 2000.

[16] H. Shimizu, Y. Sawada, and T. Sugiyama, "Minimum Tissue Size Required for Hydra Regeneration," Dev. Biol., vol. 155, no. 2, pp. 287-296, Feb. 1993.

[17] C. N. David and S. Murphy, "Characterization of interstitial stem cells in hydra by cloning," Dev. Biol., vol. 58, no. 2, pp. 372-383, Jul. 1977.

[18] C. E. AU - Juliano, H. AU - Lin, and R. E. AU - Steele, "Generation of Transgenic Hydra by Embryo Microinjection," JoVE, no. 91, p. e51888, 2014. 
[19] J. Wittlieb, K. Khalturin, J. U. Lohmann, F. Anton-Erxleben, and T. C. G. Bosch, "Transgenic Hydra allow in vivo tracking of individual stem cells during morphogenesis," Proc. Natl. Acad. Sci., vol. 103, no. 16, pp. 6208 LP - 6211, Apr. 2006.

[20] C. Dupre and R. Yuste, "Non-overlapping Neural Networks in Hydra vulgaris," Curr. Biol., vol. 27, no. 8, pp. 1085-1097, Apr. 2017.

[21] J. R. Szymanski and R. Yuste, "Mapping the Whole-Body Muscle Activity of Hydra vulgaris," Curr. Biol., vol. 29, no. 11, pp. 1807-1817.e3, Jun. 2019.

[22] S. Siebert et al., "Stem cell differentiation trajectories in Hydra resolved at single-cell resolution," Science (80-. )., vol. 365, no. 6451, p. eaav9314, Jul. 2019.

[23] Y. Noro et al., "Regionalized nervous system in Hydra and the mechanism of its development," Gene Expr. Patterns, vol. 31, pp. 42-59, Jan. 2019.

[24] R. D. Campbell, "Taxonomy of the European Hydra (Cnidaria: Hydrozoa): a re-examination of its history with emphasis on the species $\mathrm{H}$. vulgaris Pallas, $\mathrm{H}$. attenuata Pallas and $\mathrm{H}$. circumcincta Schulze," Zool. J. Linn. Soc., vol. 95, no. 3, pp. 219-244, May 2008.

[25] L. M. Passano and C. B. McCullough, "Pacemaker Hierarchies Controlling the Behaviour of Hydras," Nature, vol. 199, no. 4899, pp. 1174-1175, 1963.

[26] W. A. Kepner and D. L. Hopkins, "Reactions of Hydra to chloretone," J. Exp. Zool., vol. 38, no. 4, pp. 437-448, Jan. 1924.

[27] L. A. Schroeder and W. M. Callaghan, "Thermal tolerance and acclimation of two species of Hydra1," Limnol. Oceanogr., vol. 26, no. 4, pp. 690-696, Jul. 1981.

[28] S. O. Mast, "Reactions to temperature changes in Spirillum, Hydra, and fresh-water planarians," Am. J. Physiol. Content, vol. 10, no. 4, pp. 165-190, Dec. 1903.

[29] T. C. Bosch, S. M. Krylow, H. R. Bode, and R. E. Steele, "Thermotolerance and synthesis of heat shock proteins: these responses are present in Hydra attenuata but absent in Hydra oligactis," Proc. Natl. Acad. Sci. U. S. A., vol. 85, no. 21, pp. 7927-7931, Nov. 1988.

[30] S. Han, E. Taralova, C. Dupre, and R. Yuste, "Comprehensive machine learning analysis of Hydra behavior reveals a stable basal behavioral repertoire," Elife, vol. 7, Mar. 2018.

[31] N. B. Rushforth, A. L. Burnett, and R. Maynard, "Behavior in Hydra: Contraction Responses of Hydra pirardi to Mechanical and Light Stimuli," Science (80-. )., vol. 139, no. 3556, pp. 760 LP 761, Feb. 1963.

[32] L. M. Passano and C. B. McCullough, "Co-Ordinating Systems and Behaviour \&lt;em\&gt;In Hydra\&lt;/em\&gt;: I. Pacemaker System of the Periodic Contractions," J. Exp. Biol., vol. 41, no. 3, pp. 643 LP - 664, Sep. 1964.

[33] N. B. Rushforth, "Behavioral and electrophysiological studies of Hydra I. Analysis of contraction pulse patterns," Biol. Bull., vol. 140, no. 2, pp. 255-273, Apr. 1971.

[34] T. Nath, A. Mathis, A. C. Chen, A. Patel, M. Bethge, and M. W. Mathis, "Using DeepLabCut for 3D markerless pose estimation across species and behaviors," Nat. Protoc., vol. 14, no. 7, pp. 2152-2176, 2019.

[35] J. J. Otto and R. D. Campbell, "Tissue economics of hydra: regulation of cell cycle, animal size and development by controlled feeding rates," J. Cell Sci., vol. 28, no. 1, pp. 117 LP - 132, Dec. 1977.

[36] H. Bode, S. Berking, C. N. David, A. Gierer, H. Schaller, and E. Trenkner, "Quantitative analysis of cell types during growth and morphogenesis in Hydra," Wilhelm Roux. Arch. Entwickl. Mech. Org., vol. 171, no. 4, pp. 269-285, 1973. 
[37] A. Joseph and G. G. Turrigiano, "All for One But Not One for All: Excitatory Synaptic Scaling and Intrinsic Excitability Are Coregulated by CaMKIV, Whereas Inhibitory Synaptic Scaling Is Under Independent Control," J. Neurosci., vol. 37, no. 28, pp. 6778 LP - 6785, Jul. 2017.

[38] K. N. Badhiwala, D. L. Gonzales, D. G. Vercosa, B. W. Avants, and J. T. Robinson, "Microfluidics for electrophysiology, imaging, and behavioral analysis of: Hydra," Lab Chip, vol. 18, no. 17, pp. 2523-2539, 2018.

[39] G. Duret et al., "Magnetic Entropy as a Proposed Gating Mechanism for Magnetogenetic Ion Channels," Biophys. J., vol. 116, no. 3, pp. 454-468, Feb. 2019. 


\section{Supplemental Information}

Supplemental Figure 1: Day-to-day variance in intra-animal neural activity. a Raster plot of Hydra stimulated at $36^{\circ} \mathrm{C}$ on Day 1 and Day 3. b Calcium spike rate comparison between day 1 and day3 for individual animals (Mann-Whitney $U$ test, ns $=$ not significant, ${ }^{* * *} p<0.0001, \delta=$ Effect size using Cliff's delta)

\section{a}

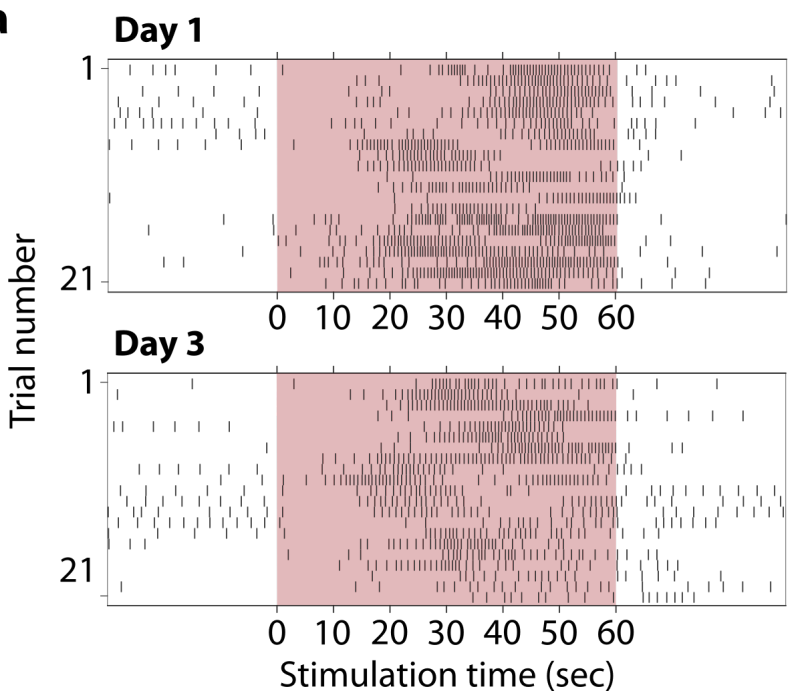

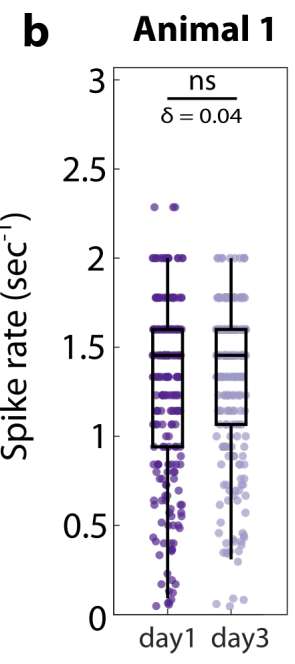
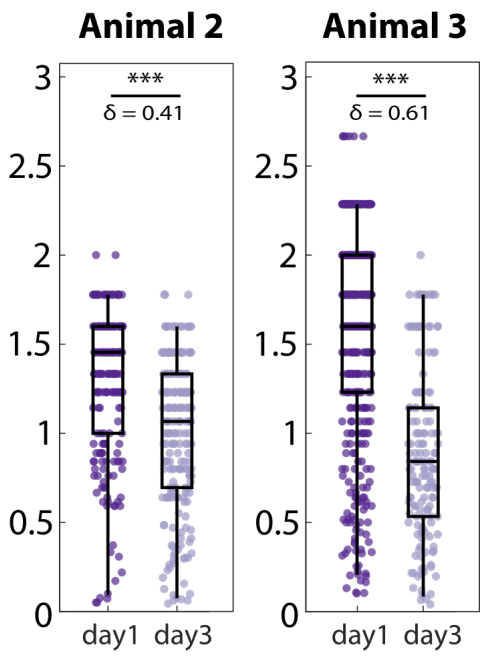

Supplemental Figure 2: Differences in neuron counts due to Hydra's natural size variations. a Size distribution of small and large Hydra. b Comparison of neuron count of small and large Hydra groups at each stimulation temperature. $(\mathrm{N}=3)$
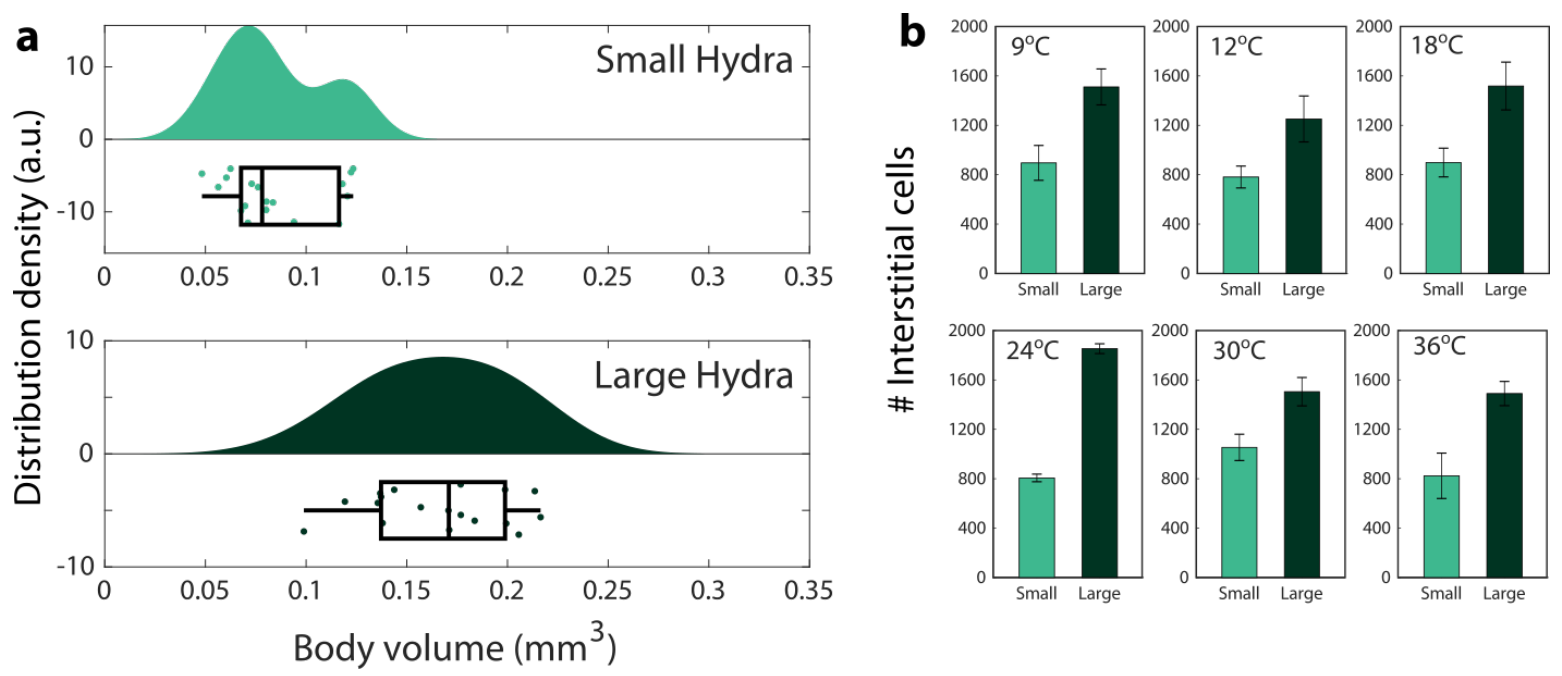

Supplemental Figure 3: Bootstrapping analysis on spike rates of whole and bisected Hydra. Each animal was stimulated 7 times in one experiment (see Materials and Methods), with 5 animals per stimulation temperature. For bootstrapping, we pooled calcium spike rates from 7 stimulation periods across the aforementioned 5 animals - at least one period from each of the animals - to create one artificial animal. Bootstrapping was done 100 times to create the calcium spike rates shown in this figure. 

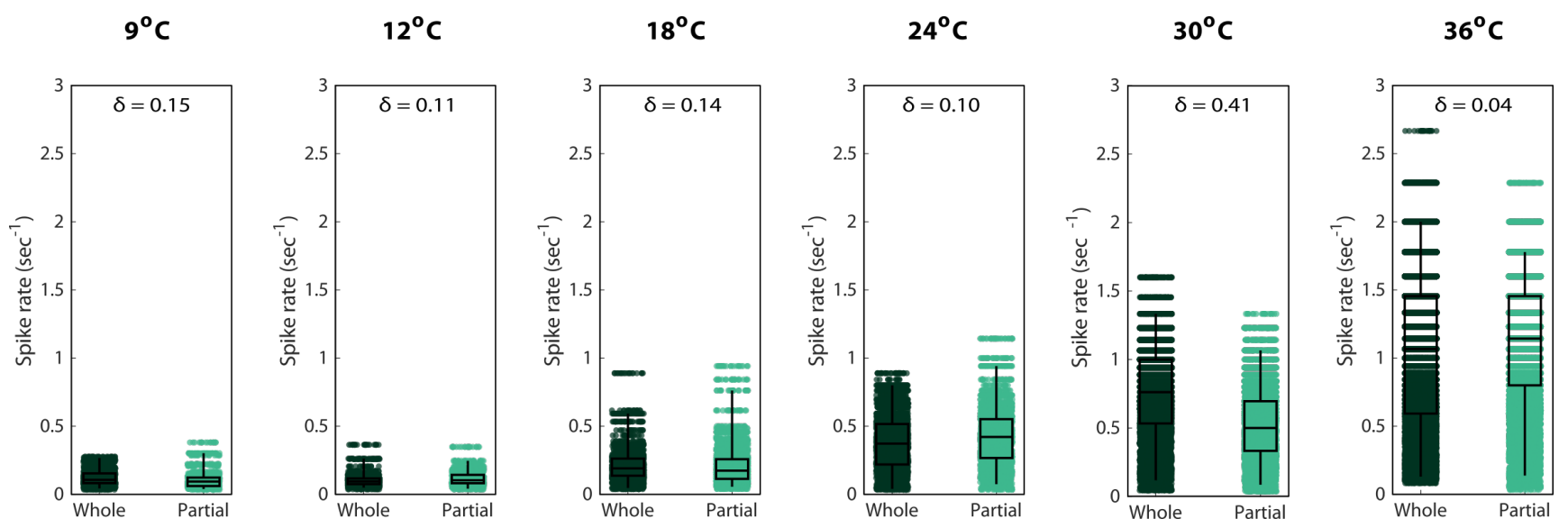

Supplemental Video 1: Hydra that was cultured at $18^{\circ} \mathrm{C}$ undergoing a thermal stimulation assay, with non-stimulus periods at $18^{\circ} \mathrm{C}$ and stimulus periods at $30^{\circ} \mathrm{C}$. The yellow trace indicates fluorescence changes in an ROI encompassing TR neurons, and orange-brown rectangles indicate stimulus periods.

Supplemental Video 2: Hydra that was cultured at $18^{\circ} \mathrm{C}$ undergoing a thermal stimulation assay, with non-stimulus periods at $18^{\circ} \mathrm{C}$ and stimulus periods at $18^{\circ} \mathrm{C}$. The yellow trace indicates fluorescence changes in an ROI encompassing TR neurons, and orange-brown rectangles indicate stimulus periods. 